\title{
Is Endoscopic Repair a Successful Method in the Treatment of CSF Rhinorrhoea? A Retrospective Study.
}

Dissanayake S., Dayasena R. P.

\begin{abstract}
\section{Background}

CSF rhinorrhoea is an important condition that can lead to morbidity and mortality. The endoscopic method has become the gold standard today for the treatment of nasal CSF fistulae. Many authors have shared their experience in endoscopic CSF leak repair but Sri Lankan data are not available. Hence, it is important to retrospectively audit the Sri Lankan practice. The study aimed at analysing the demographic data and success rates of endoscopic CSF leak repair.
\end{abstract}

\section{Methodology}

It is a retrospective study. Clinical records of all the patients who have undergone endoscopic CSF leak repair at the National Hospital of Sri Lanka during the past 7 years were included in the study.

\section{Results}

29 patients were included in the study. Whilst $20(69 \%)$ patients were having spontaneous CSF rhinorrhoea $9(31 \%)$ patients had a traumatic aetiology. Out of the patients with spontaneous CSF leak repair $16(80 \%)$ were females. The overall success rate of the surgery was $93 \%$ which was keeping the international published data.

\section{Conclusion}

Endoscopic CSF repair is a method which is globally accepted and has a very low morbidity and mortality.

\section{Introduction}

CSF rhinorrhoea is the condition where the cerebrospinal fluid leaks in to the nasal cavity through a defect in the skull base. In a normal person there is approximately $150 \mathrm{ml}$ of cerebrospinal fluid but the amount produced by choroid plexus, capillary ultrafiltration and ependymal cells are about $800 \mathrm{ml} .{ }^{[1]}$ Hence there is a very high turnover of CSF.

The incidence of CSF rhinorrhoea in Sri Lanka is not known but said to be rising in other countries especially in the United States. ${ }^{[2]}$

According to the American rhinology society the causes for CSF rhinorrhoea can be classified as Traumatic, accidental traumatic or iatrogenic, tumour related and spontaneous. ${ }^{[2]}$ Most of the scholarly articles have defined spontaneous CSF rhinorrhoea as an entity where the cause for the problem is not clear. However recently there are discussions that majority of the people classified as spontaneous CSF rhinorrhoea could be having intracranial hypertension. In Sri Lanka investigations are directed firstly to confirm the diagnosis of CSF rhinorrhoea and secondly to identify the site, size of CSF leak as well as to exclude a tumour.

The site of CSF leak is either directly on to the nasal cavity through the cribriform plate or indirectly through a sinus. Fovea ethmoidalis is the commonest site of leak followed by cribriform plate, sphenoid sinus and frontal sinus. ${ }^{[3]}$

Initially all patients who are having confirmed CSF rhinorrhoea will be given a trial with the conservative management.

Conservative management include

1. Strict bed rest

2. Advice to avoid and supportive measures to avoid coughing, sneezing and straining.

3. Carbonic Anhydrase inhibitors to reduce the production of CSF. e.g. - Acetazolamide 
Dissanayake S., Dayasena R.P. Is Endoscopic Repair a Successful Method in the Treatment of CSF Rhinorrhoea? A Retrospective Study. Volume: 6; Issue1; pp 5-11

\section{Lumbar drain}

5. Antibiotics are controversial as some argue subclinical meningitis could heal the defects in CSF rhinorrhoea.

Surgical interventions as part of the treatment for CSF rhinorrhoea is reserved for patients who do not improve with the conservative management for two weeks. There are many surgical methods available for the repair of CSF leak namely, ${ }^{[4]}$

1. Intracranial approach - Reserved for patients who have other intracranial problems that needs to be dealt at the same time. E.g. patients who have tumour related CSF rhinorrhoea.

2. Extra cranial approach - usual extra cranial approaches are extradural. e.g. repair following external etmoidectomy.

\section{Endoscopic approach}

Endoscopic approach to repair CSF rhinorrhoea is first introduced by a surgeon called Wigand in 1981. [5] Since then the use of endoscopes in repair of CSF rhinorrhoea has evolved and today considered the gold standard. It has many advantages with a very low morbidity and mortality.

In Sri Lanka endoscopic repair has become a challenge due to limited endoscopic facilities at hospitals. Even if the best endoscopes are available skull base repair needs lot of skills and hands on experience. Hence it is difficult to gather a large series of patient who have undergone endoscopic CSF repair in Sri Lanka. The study carefully audits the endoscopic repair done for CSF rhinorrhoea by the second author from the year 2009 to 2015 at the National Hospital of Sri Lanka.

\section{Aim}

The main aim of the study is to identify the demographic features, site of leak, success rate and complications of surgery among the patients who underwent endoscopic repair of CSF rhinorrhoea.

\section{Methodology}

This is a retrospective study auditing the surgical outcome of the patients who have undergone endoscopic CSF rhinorrhoea repair at the National Hospital of Sri Lanka. Demographic data were obtained from the operation register and the ward admission books. The possible aetiology was recorded from the clinical history in the patient in ward records. The site of defect in the skull base was noted from the intraoperative notes. The success of the surgery was determined solely on the absence of CSF leak at the end of three months. The clinic records were obtained to determine the success rate and the complications of the surgery. Due to incomplete recording the study mainly looked at the primary complications.

Firstly, the Bed Head Ticket (BHT) numbers of all the patients who have undergone endoscopic repair for CSF rhinorrhoea were obtained from the theatre surgical registry. Secondly, the inward admission records were traced from the medical records office and necessary information was gathered. Thirdly the ward admission books were used to obtain the clinic record numbers. However, some of the cases were removed from the series due to the unavailability of the clinical records thence success rate and complications.

All the surgeries in this series were not done as planned elective surgeries. Some were done as 'emergencies' when the patients were admitted with complications such as meningitis. Patients who have complained of watery nasal discharge have had testing of the discharge for sugar. The diagnosis of CSF rhinorrhoea was made if the sample is colourless and the glucose level is more than $50 \%$ of the blood glucose value. Testing the sample for Beta transferrin protein was not available in the NHSL.

With a presumptive diagnosis of CSF rhinorrhoea from the above testing, patients underwent CT cisternograms. MRI scan was not performed as part of the perioperative work up in all patients.

Informed written consent had been obtained and the other necessary haematological and radiological investigations to assess the fitness 
for surgery had been done. Antibiotics had not been given routinely unless the patient was having an episode of meningitis. Once the fully consented prepared patient is taken to theatre a general anaesthetic had been given. A single dose of antibiotics had been given at the time of induction. Abdominal fat and External oblique aponeurosis was taken as graft material. Lumbar puncture and injection with dye to identify the defect had been used in patients where the defect was uncertain on CT cisternogram.

Prior to initiation of surgery the nasal cavities were decongested. Following decongestion examination of the lateral wall was done. When necessary a limited septoplasty had been done to gain access. Secondly, a middle meatal antrostomy followed by an anterior and posterior ethmoidectomy had been done. (During examination if a clear site of the defect was found in the cribriform plate sinus antrostomies were not done.) Sphenoidotomy was done when the skull base defect was in the sphenoid sinus.

The Surgical technique was 'the underlay method' in all patients. Once the defect is found the dural sac is

separated from the surrounding and everted back. Then the defect in the bone is filled with fat graft. On the fat graft fascial graft is laid and often another fat tissue layer and absorbable gelatin layer is placed to further support the fascial graft. When necessary mucosal flaps from the turbinates were also used.

\section{Results}

There were a total number of 38 patients who have undergone endoscopic repair for CSF rhinorrhoea at the National hospital of Sri Lanka from 2009 to 2015 . Due to the practical inability to trace the clinic records and/or incomplete recording, only 29 patients were included in the study.

Out of 29 patients 19 patients (65\%) were females and $10(35 \%)$ patients were males.

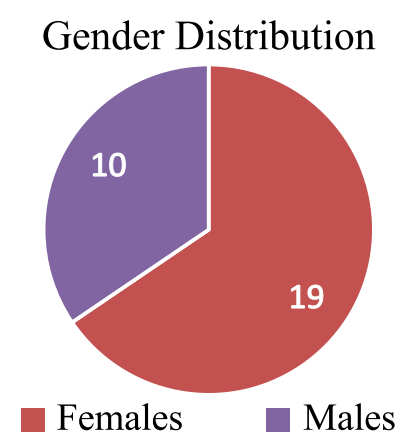

Chart 1 - Gender Distribution

The right side was involved in $14(48.3 \%)$ patients and the left side was involved in $15(51.7 \%)$ patients. The Age distribution was from 21 years to 62 years and the mean was 41.72 . In males the mean was 39.4 yrs. and in females the mean age was 42.95 yrs. A total of 9 (31\%) patients had a history of trauma and the remaining $20(69 \%)$ patients had spontaneous CSF rhinorrhoea. There were three patients following iatrogenic trauma of which one patient had developed CSF rhinorrhoea following craniotomy done to resect a malignant tumour. In the subgroup with spontaneous CSF rhinorrhoea 16 (80\%) were females and $4(20 \%)$ were males.

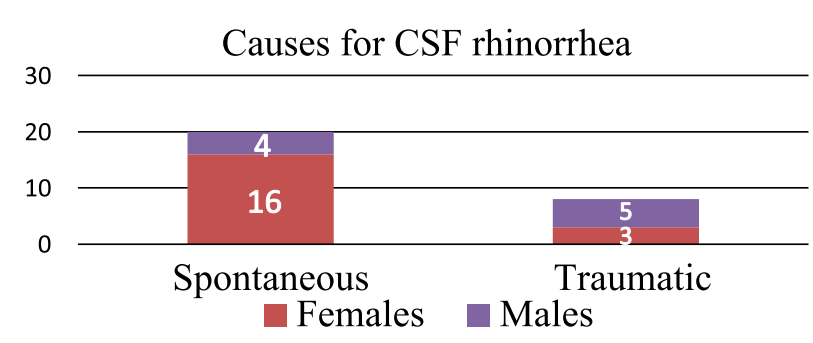

Chart 2-Causes of CSF rhinorrhoea with

gender distribution

Meningitis is known to be associated with CSF rhinorrhoea. Out of the 29 patients 10 (34.5\%) patients had a prior history of meningitis. In some patients' recurrent meningitis was the main presenting complaint. 3 patients had undergone endoscopic repair while on antibiotic treatment for meningitis.

Out of the chronic medical conditions commonly seen in the general population incidence of chronic essential hypertension, Bronchial asthma and 
Diabetes mellitus was looked at among patients with spontaneous CSF rhinorrhoea. A total of $12(60 \%)$ out of 20 patients had hypertension, 4 $(20 \%)$ patients had bronchial asthma and $4(20 \%)$ patients had Diabetes mellitus.

The site of leak and the defect in the skull base was taken according to the surgical notes irrespective of the site of leak reported in the CT cisternogram. 11 (37.9\%) patients each were found to have defects in the cribriform plate and sphenoid sinus. In 4 patients $(13.79 \%)$ the defect was in the fovea ethmoidalis and in $1(3.45 \%)$ patient each the defect was in the sphenoethmoid recess and the frontal sinus.

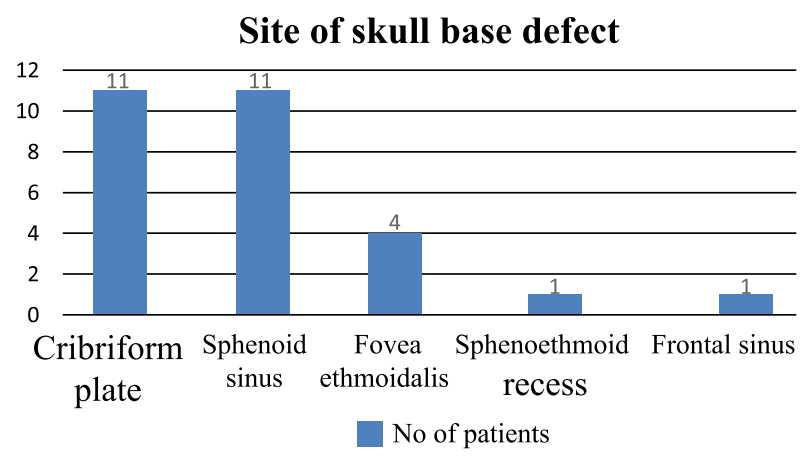

Chart 3 - Site of the skull defect

In $3(6.9 \%)$ patients out of 29 patients CSF rhinorrhoea had recurred following surgery. However, in one patient this had settled with the conservative management, one required repeat surgery and the other patient defaulted treatment. Hence the success rate at the end of one surgical endoscopic repair is $93 \%$.

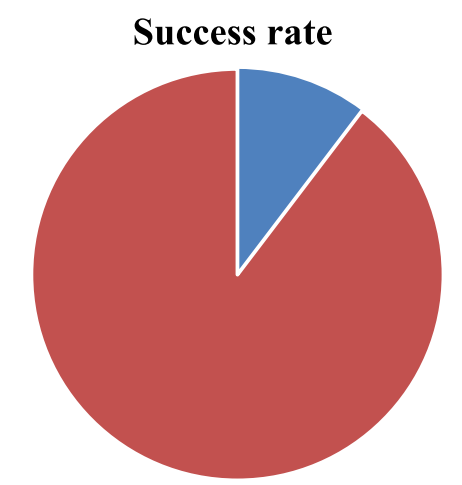

Recurrence $\square$ No recurrence

Chart 4 - Success rate at the end of 3 months.
One patient developed a serious complication following surgery which is loss of vision in one eye. According to expert opinion there had been evidence of increased pressure on the optic nerve. This patient had a history of malignancy with radio irradiation of the skull thus the complication could be multifactorial.

\section{Discussion}

CSF fistulae is a troublesome condition as it creates a communication between the cranial cavity and the exterior. Nowadays CSF fistulae are said to be of rising incidence and one study has shown that there is a twofold rise in the incidence of CSF rhinorrhoea in United Kingdom from 2002 to $2012^{[6]}$ This could be partly due to the increasing awareness among the medical professionals.

From an academic point of view CSF fistulae can be categorized in to normal pressure and high pressure. Normal pressure fistulae can be further classified in to spontaneous and traumatic. High pressure fistulae are clinically difficult to diagnose unless associated with features such as headache, vomiting, weakness, lethargy together with other neurological signs. Hence, intracranial hypertension in early stages can be missed. ${ }^{[7]} \mathrm{CT} /$ MRI has an additional benefit in patients with CSF fistulae as it could not only show the site of skull defect but also signs of raised intracranial pressure such as 'empty sella', flattened gyri, increased sulci etc., ${ }^{[8]}$ In this series none of the patients had a measurement of CSF pressure due to the lack of facilities but, none of the patients had features in the imaging to suggest intracranial hypertension. In this series majority (69\%) had spontaneous CSF rhinorrhoea and this is similar to a case series in India ${ }^{[9]}$. In a case series done in the Middle East it was shown that only 55\% of CSF rhinorrhoea was due to non-traumatic causes. ${ }^{[10]}$. Most of the literature describes that spontaneous CSF rhinorrhoea is commoner in females than in males and this study supports this statement $(80 \%)$. ${ }^{[9]}$

One of the challenges in the pre-operative work up in patients with CSF rhinorrhoea was to identify 
the site of the leak. In this series two methods were utilized to overcome this challenge. Firstly, all patients had a CT Cisternograms prior to surgery. In this study sensitivity and specificity of CT cisternogram in identifying the site of leak is not assessed. In Jeffrey et al. different modalities of imaging used to detect the bony defect of CSF rhinorrhoea are studied and HRCT had the highest sensitivity $(71 \%){ }^{[11]}$. Further studies are needed in Sri Lanka to assess the sensitivity as well as to evaluate the cost effectiveness of performing HRCT on all patients with CSF rhinorrhoea.

The commonest site of leak was the cribriform plate and the sphenoid sinus (37.9\% each). The study done by Paolo et al. describes the cribriform plate as the commonest site of leak but in his study sphenoid sinus defects were only $10 \%$. ${ }^{[12]}$ Whereas, other studies show that the fovea ethmoidalis is the commonest site of leak. ${ }^{[13]}$

Bacterial meningitis is a life threatening complication of CSF rhinorrhoea. Recurrent meningitis can be the presenting complaint as well a complication following surgery. $34.5 \%$ of the patients had at least a single episode of meningitis. Subclinical meningitis is a controversial area in CSF fistulae. Some authors argue that subclinical meningitis could be beneficial for patients with CSF fistulae as inflamed meninges may seal the site of defect. This is another argument against prescribing prophylactic antibiotics. ${ }^{[14]}$

Intrathecal fluorescin at the time of surgery is preferred by many authors. [15] Some studies show the disadvantages and the complications associated with intrathecal fluorescin injection such as seizures ${ }^{[16]}$ but, in our series none of the patients who had fluorescin injections reported any adverse effects.

There isn't sufficient data to support a direct link between common comorbid illnesses and CSF fistulae but chronic cough was identified in a study done in Iran. ${ }^{[17]}$ Vascular compression in chronic essential hypertension is known to cause intracranial complications ${ }^{[18]}$ and this could be the pathophysiology behind the association of CSF fistulae and chronic hypertension.
There are many surgical techniques described for endoscopic repair of CSF fistulae. The 'Bath plug technique' is a widely accepted technique. There are many graft materials used to seal the defect. Wigand et al describe the first free tissue grafts for CSF leak repair. Burns et al describes the use of middle turbinate mucosa as free tissue grafts and success rate was $94 \%$. Next step is the use of mucoperichondrial or mucoperiosteal flap. Lanza et al has used a combination of flaps and shown a success rate of $89 \%$. Hydroxy appetite or bone cement is also used in certain studies but its tendency to get washed off especially in high flow fistulae is a disadvantage. Bone cement can also get fractured thus creating recurrence of symptoms. Depending on the site, size of the defect, bony or cartilage 'free grafts' or flaps are used but a study that compares the success rates based on the type of graft used was not found at literature review.

In this series a combination of free grafts and flaps were used. External oblique aponeurosis and fat were used as free grafts and when necessary local flaps such as mucoperiosteal flaps from the middle turbinate were attempted. Even though the underlay technique is more technically challenging in all patients this technique was preferred. One of the features of the underlay technique is to have a good buttress around the skull defect to place the graft. This becomes difficult especially in the sphenoid sinus due to the limited accessibility hence most of the authors have used a combination of underlay and onlay techniques. Despite the technical difficulty we managed to use the underlay technique in this series.

This study has shown a 93\% success rate in endoscopic repair for CSF rhinorrhoea. Schoentgen $\mathrm{C}$ et al reports a $22.5 \%$ failure rate ${ }^{[19]}$ and Virk JS et al reports an $11 \%$ failure rate. ${ }^{[20]}$ American college recommends that the success rate of endoscopic repair should be above $90 \%$. A similar study done by Chaabal et al have shown that their success rate is $93 \%$. Hence, the overall success rates are keeping with the international standards. 


\section{Conclusion}

Endoscopic repair is a successful method of treatment for the patients with CSF rhinorrhoea in Sri Lanka.

\section{References}

1. Burt AM. Textbook of Neuroanatomy. Philadelphia: W.B.Saunders Co, 1993.

2. Nelson RF, Gantz BJ, Hansen MR .The rising incidence of spontaneous cerebrospinal fluid leaks in the United States and the association with obesity and obstructive sleep apnea. 2015Otol Neurotol. 2015 Mar;36(3):476-80

3. Kevin C. Welch MD, CSF leak. http://care. american-rhinologic.org/csf_leaks

4. Badr Eldin Mostafa, Ahmed Khafagi, Combined HRCT and MRI in the Detection of CSF Rhinorrhea, 2004,Skull Base. 2004 Aug; 14(3): 157-162.

5. Kevin C, Arlen D Meyers, CSF rhinorhea http://emedicine.medscape.com/ article/861126-overview\#a5

6. Nelson RF, Gantz BJ, Hansen MR. The rising incidence of spontaneous cerebrospinal fluid leaks in the United States and the association with obesity and obstructive sleep apnea. Otol Neurotol Mar :36(3):476-80;2015

7. https://medlineplus.gov/ency/ article/000793.htm, intracranial hypertension,medline plus

8. Schlosser RJ, Bolger WE, Significance of Empty sella cerecrospinal fluid leaks, Otolaryngol Head and Neck Surg, Jan;128(1):32-8; 2003
9. Arun A, Mathew J, Vargese AM, Ganesan S, Endoscopic repair of CSF fistulae A ten year experience ,Journal of Clinical and Diagnostic Research 10(8): MC01-MC04; 2016

10. Saafan ME, Albirmawy OA, Tomoum MO. Sandwich grafting technique for endoscopic endonasal repair of cerebrospinal fluid rhinorrhoea. Eur Arch Otorhinolaryngol. 2014;271(5):1073-79. [PubMed]

11. Stonea JA, Castillea M, Neelona B, Mukherjia S. 1999, Evaluation of CSF leaks : High Resolution CT compared with Contrast enhances CT and Radionuclide Cisternography, American journal in Neuroradiology, 1999 20: 706-712.

12. Castelnuovo P, Mauri S, Locatelli D, Emanuelli E, Delù G, Giulio GD. Endoscopic repair of cerebrospinal fluid rhinorrhea: learning from our failures. Am J Rhinol. 2001;15(5):333-42. [PubMed]

13. Mostafa BE, Khafagi A, 2004, Combined HRCT and MRI in the detection of CSF rhinorrhea, Skull base 2004Aug; 14(3): 157-162

14. Spontaneous CSF rhinorrhea:Scott brown Vol ;2007

15. Zweig JL, Carrau RL, Celin SE, Schaitkin BM, Pollice PA, Snyderman CH. The use of low- dose intrathecal fluorescein in endoscopic repair of cerebrospinal fluid rhinorrhea. Arch Iran Med. 2013;16(5):264-66. [PubMed] 
16. Presutti L, Mattioli F, Villari D, Marchioni D, Alicandri-Ciufelli M. Transnasal endoscopic treatment of cerebrospinal fluid leak: 17 years' experience. Acta Otorhinolaryngol Ital. 2009;29:191-96. [PMC free article] [PubMed]

17. Mishra KM et al. 2016, Endoscopic repair of CSF rhinorrhea : An Institutional experience , Iranian Journal of Otorhinolaryngology, Jan; 28(84):39-43

18. Jannetta PJ, 1980, Neurovascular compression in cranial nerve and systemic disease, Annals of Surgery Oct ; 192(4):518-525

19. Schoentgen C, Henaux PL, Godey B, Jegoux F. Management of post-traumatic cerebrospinal fluid (CSF) leak of anterior skull base: 10 years experience. Acta Otolaryngol. 2013;133(9):944-50. [PubMed]
20. Virk JS, Elmiyeh B, Stamatoglou C, Saleh HA. Optimising outcomes in the management of spontaneous cerebrospinal fluid rhinorrhoea. Rhinology. 2013;51(3):268-74. [PubMed]

21. Chaaban MR, Illing E, Riley KO, Woodworth BA. Spontaneous cerebrospinal fluid leak repair: a five-year prospective evaluation. Laryngoscope. 2014;124(1):70-75. [PubMed] 